\title{
Continual Business Transformation Technology
}

\author{
Thomas Li, Wei Ding, Chunhua Tian, Rongzeng Cao, Shunxiang Yang, and \\ Jun Zhu \\ IBM China Research Lab, \\ Beijing 100094, China \\ \{thomasli, dingw, chtian, caorongz, yangsx, zhujun\}@cn.ibm.com
}

\begin{abstract}
Enterprises always have to pursue for profitability while maintaining long term competitive advantages. Today, this task becomes more challenging due to globalization and dynamic changing business environment. For each enterprise, this task can not be fulfilled without changes to its business infrastructure and IT systems. That is, enterprises need to transform themselves to adapt to both external and internal changes. This kind of transformation is not simply any transactional act, but rather a never-easy journey. In this paper, the concept of continual business transformation is introduced with key challenges identified. Based on experience from practical cases, we propose a set of business transformation technology framework that incorporates component-based approach and business-driven architecture. In the framework, $C B M$ (Component Business Modeling) method is to identify transformation opportunities and provide guidance to IT architecture design from business perspectives. As transformation needs to be conducted in a holistic view, IT Blueprint in the framework is to provide an enterprise-wide IT design and governance model. To avoid building more silo applications as happening today in many cases, the IT Blueprint can be used to guide solution development for better alignment among different applications as well as across various stages during the transformation. Another keystone in the framework is Assetization, which is proposed for quality improvement and cost reduction from harvesting and reusing artifacts built in the transformation journey. Furthermore, we also illustrate how these methods and technologies were validated in a transformation engagement with a leading international bank in Asia-Pacific with satisfactory results.
\end{abstract}

Please use the following format when citing this chapter:

Li, T., Ding, W., Tian, C., Cao, R., Yang, S., Zhu, J., 2006, in International Federation for Information Processing, Volume 205, Research and Practical Issues of Enterprise Information Systems, eds. Tjoa, A.M., Xu, L., Chaudhry, S., (Boston:Springer), pp.85-95. 


\section{Introduction}

In today's dynamic business environments, enterprises are forced to change in a much faster pace and broader scope [1]. Due to the limitation of resources (e.g., budget, time, human resource, etc.), concerns of risk factors, and other organizational issues, enterprises typically can not accomplish their intent in a single act. In many cases, there are changes upon the results of previous changes. Looking from a holistic view, these changes require a conscious design, instead of relying on any random, impulsive, and reactive action. These changes, when executed under a grand plan, form the transformation of the business. Transformation is a long journey, not a one-shot transaction. It has to be carried out in an incremental and progressive fashion. Each project in transformation has to be leverageable to justify succeeding projects with sustainability. Therefore, we propose this notion as "continual business transformation" and focus our research on developing innovative methods and tools to ensure coherent transformation across the whole transformation lifecycle.

The rest of this paper is structured as follows. In Section 2, business transformation trends are discussed and the gaps in methodology are examined. Then a business driven end-to-end transformation methodology framework is presented in Section 3. Its application in a customer engagement is presented in Section 4. Concluding remarks are presented in Section 5.

\section{Continual Business Transformation}

The environment of enterprises is under changing. Demand becomes more dynamic. Competition is more severe. New business models are continually emerging. Enterprises need transform their business model and operations, with prompt and strong supports from corresponding IT systems, so that their businesses can keep up with the demands from both the environment and their own strategies [2]. The "perfect" state of business transformation is that an enterprise can respond with speed to any customer demand, market opportunity or external threat with an integrated end-to-end business process across the company and with key partners, suppliers and customers.

It may look like a simple task superficially. In many of the state-of-art practices, practitioners believe that the final $\mathrm{To}-\mathrm{Be}$ state of a business and its IT is most critical. The typical practice will compare the To-Be against the $A s-I s$ state, identify the gap, define the transformation roadmap accordingly, execute, and it's done! But realities suggest differently.

First, business transformation takes time and things change. During the journey, the whole ecosystem that affects the enterprise will not remain static. There are always significant changes beyond control. This makes the ideal To-Be state constantly changing as a moving target in the dynamically changing business environment. This concept is illustrated in Fig. 1.

Second, business transformation is carried out under constraints. With pressures from many dimensions, transformation cost and resource allocation require strong justification all the time. All these factors make the business transformation 
extremely hard in real practice, especially for the businesses with strong awareness of ROI (Return of Investment), time-to-market, and sustainable growth.

Third, business transformation often requires preparation in many aspects, both business side and IT side. Each side alone is incapable of accomplishing the targets.

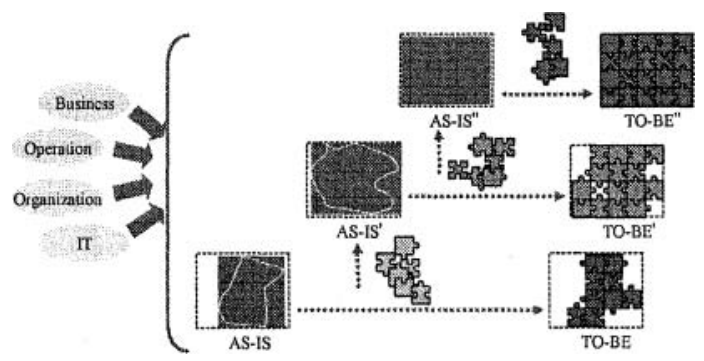

Fig. 1. Continual Business Transformation

For the moving target - $\mathrm{To}-\mathrm{Be}$ state, it is important to follow a robust and systematic methodology to transform their businesses and building their IT system. Then all the artifacts generated each time by the methodology have some characteristics even the view of the future is evolving. Meanwhile transformation needs to take an incremental and progressive approach. It can not be done in one shot, but through a long journey.

Reducing cost and risk while achieving more is very important for sustainable transformation. This can be achieved via asset reusing, better communication for consensus, and by leveraging industry best practices. To achieve high levels of reuse, asset need be organized in a reusable way, i.e. well aligned with business and IT architecture. Reuse is not a separated process or project; instead it should an integrated part of the transformation journey [3].

In information technology, component paradigm and architecture is an efficient way to enable applications mapping on various execution platforms and provide adaptability $[4,5]$. Since business changes are often the source of IT system changes, business is also desired to be componentized to speed up the responses speed to market changes and keep IT changes at the reasonable level.

These several factors and capabilities, which are critical to the success of transformation, are the ones in the journey of what we call "Continual Business Transformation". The "continual" not only reflects in continually changing environment and business needs, but also the evolving transformation journey. 


\section{An End-to-End Business Transformation Methodology}

\subsection{Methodology Overview}

Based on our study and practice, a Continual Business Transformation (CBT) methodology is formed and proposed to facilitate the end-to-end transformation from business strategy to solution development as shown in Fig. 2.

- At business side, CBM (Component Business Modeling) method is adopted to componentized business to provide guidance to IT architecture design, and identify transformation opportunities as well as develop business transformation roadmap.

- At technology side, IT Blueprint method is addressed to build the enterprise-wide architectures to guide solution level design to ensure the consistency and benefit the enterprise as a whole.

- At business operation and solution side, modeling technologies in BPM (Business Process Management) is introduced to support and link the enterprise level and solution level designs (including both business and IT). Modeling technologies and tools is applied to build component-based IT solution design/implementation to fulfill the enterprise transformation vision.

- Asset Management technology is adopted to accelerate the continual process by asset harvest and reuse.

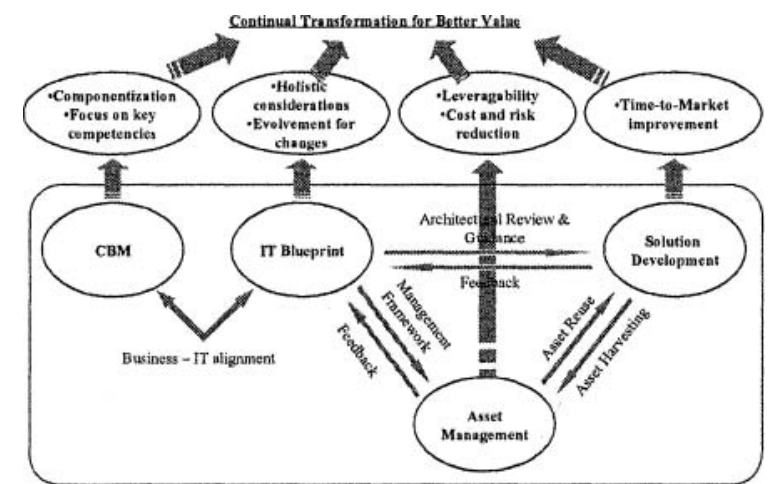

Fig. 2. Continual Business Transformation Framework Overview

Since CBT methodology is a systematic end-to-end methodology, from business level to IT level, the linkage between different parts also has been formalized and structured for interactions. Based on the integrated framework and formalized models, the requirements mentioned in Section 2.2 can be well satisfied. It should be emphasized that the end-to-end methodology does not mean that each practice should begin from CBM, then to IT blueprint and finally to solution development. In fact, it can start from any parts according to the real situation. In the followings sections, the detailed technologies for each part will be introduced. 


\subsection{Business Component Modeling}

The idea of viewing a business as a set of interlocking components - and the underlying notions of specialization and reusability - isn't exactly new $[6,7]$. Businesses have always understood that to be as efficient, effective, and profitable as possible comprising components with discrete services. Yet traditional business modeling, based on mapping individual business processes, does not incorporate this idea. Component business modeling (CBM), which is a business decomposition methodology that has been used successfully by IBM in various engagements across industry sectors $[8,9]$, allows analysis from multiple perspectives because it groups like activities together without regard to organizational, geographic or process boundaries, companies can more readily spot redundancy - similar business resources (people, assets and technology) that are duplicated in other corners of the company, as well as strategic, differentiating capabilities.

CBM offers a new way to analyze business and enables a clear focus on the strategic capabilities of the business. And, applying various evaluation criteria to the components-e.g. cost, revenue, strategic fit-produces a 'Heat Map,' which shows where opportunities exist. Figure 3 shows an example of a component business model for the banking industry.

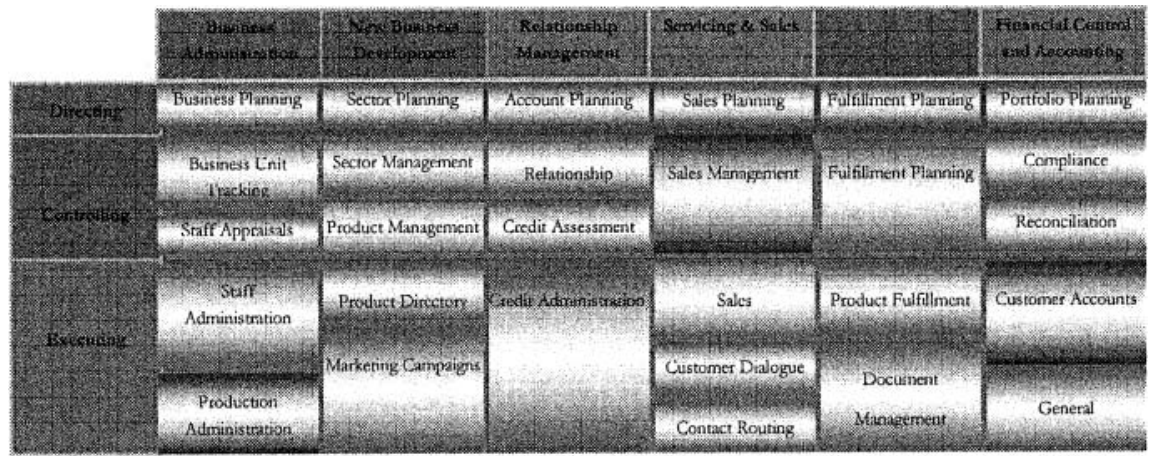

Fig. 3. A component business model

Further, we can model $A s-I S$ state and $T o-B e$ state separately with the same structure. To-Be state modeling can leverage industry map comprising best practices. Based on the comparison with $A s-I s$ model and TO-Be model, the gaps can be identified for transformation opportunity generation. The gap analysis of the business component efficiency and effectiveness determines if certain technology-based reengineering can help streamline the process, improve operating efficiency and optimize strategic business advantage. Generally, three types of issues tend to arise in the shortfall assessment:

- A structure gap is a qualitative statement of the shortfall between current business organization and those that were best practices.

- A performance gap is a quantified statement of the shortfall between current 
business results and those that were expected.

- An opportunity gap is a quantified assessment of the discrepancy between current business results and those achievable with a new business design.

While a performance gap can often be closed by focusing on superior execution, with no change to business design, and structure gap can be closed by simple business design, closing an opportunity gap requires a new business design.

\subsection{IT Blueprint}

As CBM provides a good base for figuring out the appropriate business model for an enterprise, analyzing the business operations and identifying the business transformation opportunities, we also need similar holistic views for IT. These holistic views can provide a base for individual pieces of the IT to be well described and planned, with guidelines and principles defined which can help govern future actions on refining the holistic views and the individual pieces. Although these views enforce some kind of constraints on individual pieces and individual transformation projects, it helps avoid creating new silos which can not talk with each other, and benefits the whole continual transformation a lot. We call these holistic views IT Blueprint as a whole, and have defined a framework to systematically organize the views in a way so that they can be better described, managed and used in guiding the IT transformations. Figure 4 illustrates the high level structure of the framework.

As the core of an IT Blueprint, the Blueprint Model describes the total picture of the enterprise's IT from Application, Data and Technology perspectives. This separation of concerns is crucial, because to align IT with business, we must identify what pieces of the IT are most relevant to business operations, and what the interrelationships are. Based on our study, applications and data are very relevant ones while technologies focus more on infrastructure, which can be business function neutral to a certain extent. According to best practices nowadays, applications should be componentized and align with business operations, for which the Business Components in CBM can provide good guidance on defining the boundaries for application level components. Data should be defined by taking into account the business information needs at business operation level. In addition to the core models, principles, guidelines and standards need to be defined, together with a governance framework, which includes the necessary organizations and processes, to help govern the future changes of the core models and the execution of individual IT transformation projects. Also, according to the specific requirements of the enterprise, the suitable reference architectures can be selected and customized, which can be used as architectural assets for reuse to guide high level designs of individual solutions. 


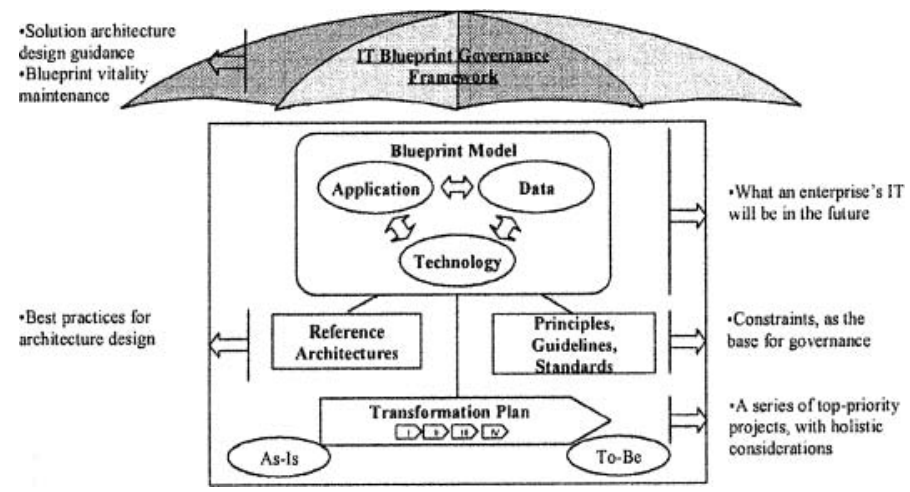

Fig. 4. IT Blueprint Framework

Although IT Blueprint is important, its value seems not directly related to short term successes. Meanwhile, building a complete IT Blueprint is costly in terms of time, money and skills involved. Even worse, the value of the static To-Be IT Blueprint built through months or even years is often under suspicion, because during that period of time, a lot of changes will happen and the To-Be can be quite different. That is why resources and supports usually go to urgent projects like adding new functions of strong customer demands or resolving a performance problem which customers complain a lot. That is also why in the long run, without architectural guidance and governance, enterprises usually face a lot of problems and come to realize that IT Blueprint is the key for a successful continual transformation.

\subsection{Solution Development}

IT solution building has always been the key step for enterprise business transformation. It is through solution building that business/IT strategic planning and analysis finally get into reality. With the business environment gets more and more complex \& dynamic, which in turn generates higher and higher expectation on IT technologies from business side, the traditional way of building IT solutions are becoming less competent. The following challenges can be easily observed in today's solution building practices [10]:

- Gap between business requirements and IT capabilities

- Gap between overall IT architecture and individual IT solutions

- Gap on IT skills and business knowledge

Model-driven solution building approach is adopted in the methodology to address all above challenges. Basically, this method is built up by referencing and enhancing existing methodologies (such as Rational Unified Process). Figure 5 shows the main development phases and key features in the method. Models can help users better communicate with each other to gain better understanding of requirements, to design well structured solutions, and to maintain and manage the biway linkage between them. With the modeling of overall IT blueprint, adding 
corresponding solution building technologies that can fully leverage them, we can manage to build solutions that are in align with the overall blueprint, which are also evolving by receiving input from each single solutions. For more information, please refer [10].

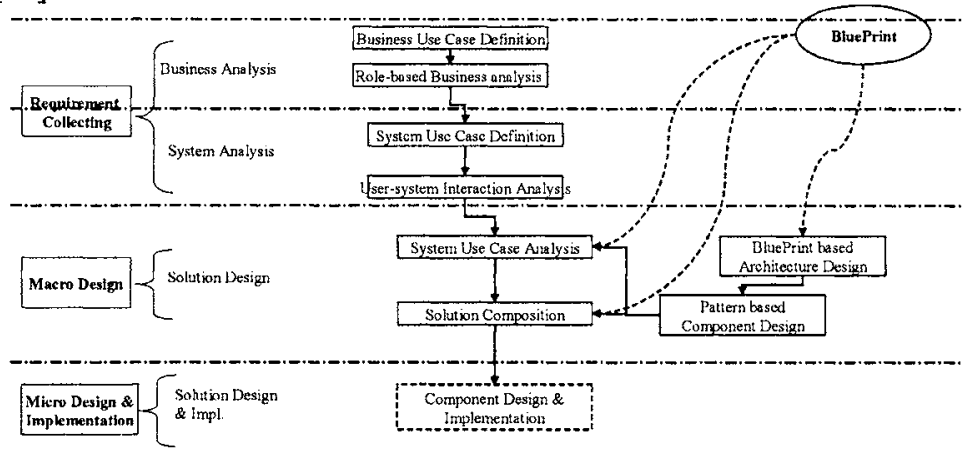

Fig. 5. Solution Building Methodology

\subsection{Assetization}

Sometimes the value of assetization of the business and IT stuff for a single client is not fully realized, because people usually think the chances of reuse are quite limited (although some clients need to replicate certain solutions across various geographies, and some even want to sell their own solutions to others). But if we take the time dimension of the continual transformation and the changes during the course into consideration, assetization shows specific value - once the key knowledge of both the business operations and IT systems are captured in appropriate formats, packaged and managed as assets across the enterprise, you can find that some inhibitors for continual transformation disappear. For example, the artifacts of previous solutions can be easier to share, understand and leverage in follow-on projects to facilitate decision making and development, and the strong dependence on certain key people can be alleviated to reduce cost and risk, etc.

Assets can be harvested and reused in the end to end methodology, from CBM model, IT blueprint, to solution components and infrastructure patterns. The two natures of asset are value and reusability. To increase the reusability, there are three key perspectives for asset based development as shown in Figure 6. 


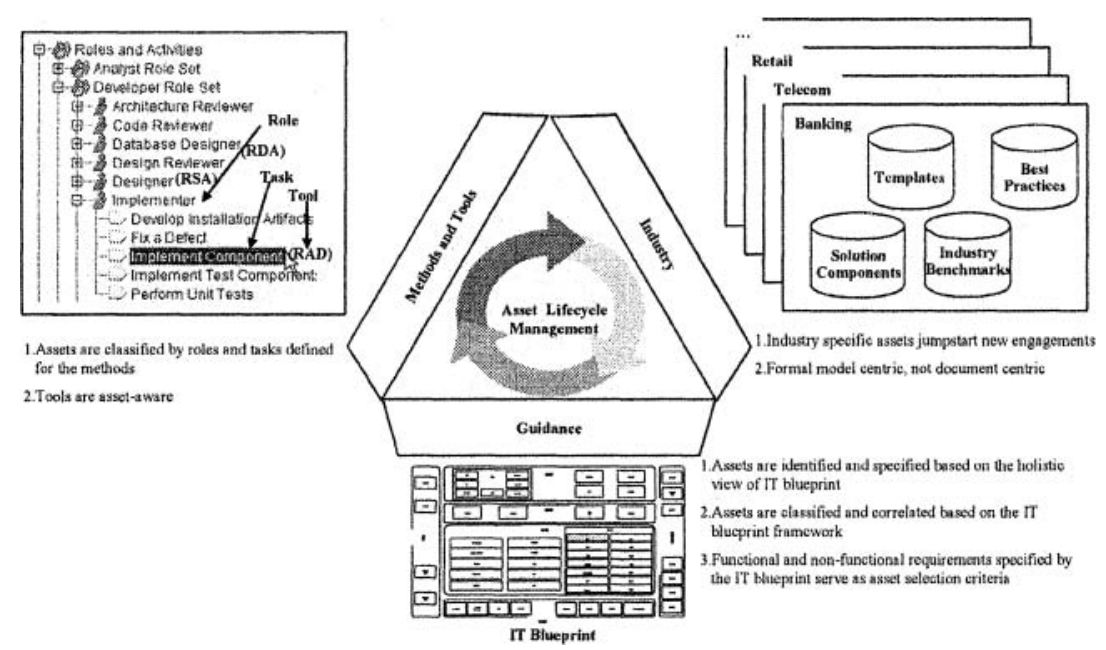

Fig. 6. Assetization in continual business transformation

- Assets should be integrated with solution methodologies and tools. Facilitated by asset aware method and tools, solution developers find right assets at right stage of methodology; at the same time, solution artifacts are developed not only for individual solution, but also developed for reuse.

- Assets can be better planned, organized and managed by using some holistic views of the enterprise, for example, IT Blueprint is good for managing IT assets. Its contents (function specification, non functional requirements, principles ...) can be served as important guidance for assetization, to improve consistency, reliability and quality. Meanwhile, since we follow IT Blueprint guided approach for solution development, the well-defined structure and relationship between IT Blueprint and IT assets can help us to clearly identify the assets that can be reused, need to be enhanced or developed in an individual solution. Similar to what IT Blueprint does for IT assets, CBM views, on the other hand, provide a good base for organizing knowledge and designs of business functions, processes, metrics, etc.

- Assets should be industry or domain specific to formally represent industry or domain best practices and insights.

\subsection{Other Key Technologies}

In the above framework, there is many types of models covering different perspectives and are closely related with each other. A common environment for model linkage is very important. Layered models play crucial roles in model driven business transformation. Models are prone to be changed due to various reasons. Our practice reveals that frequent model changing results in a big issue - inconsistency is usually brought by and conflict between models gradually erodes the overall 
solution. Current manual method costs too much and the veracity of conflict analysis is doubtful. Therefore, systematic technologies are needed to help tracing the related models in a solution, analyzing the propagation of change impacts and synchronizing the impacted models to keep the overall consistence of the solution.

In enterprise modeling, there are a lot of glossaries. It is indispensable for effective business analyzer to have a clear semantic relationship between them. So semantics technology is very important.

\section{Case Study}

The methods and technologies illustrated in this paper were validated in a transformation engagement in a leading international bank in Asia-Pacific. The bank mission is to become a trusted financial institution of the Pacific Rim. In the past years, this bank has achieved numerous accolades from leading financial periodicals for its competitive capability and innovation.

In an industry transformed by deregulation and consolidation, this bank is facing increasingly intense competition that forces them to find ways to increase revenue and improve customer satisfaction. Facing these challenges, this bank wants to do the following transformations: 1) enhancing strength in potential $M \& A ; 2$ ) core System Transformation to support continual improvement to sustain competency in market, and 3) being Customer Centricity to explore cross-sell and up-sell opportunities to increase profit margin. In 2004, facing market pressure and potential M\&A opportunity, this bank wanted a more strategic change on core banking system and business operation. Thus Customer Oriented Core Banking System Transformation project was kicked off. On June 2005, IBM China Research Laboratory delivered a continual business transformation method to help this bank transform its flagship financial product from their legacy system to a new open framework, which is componentized, scalable, flexible and easy to reuse in future. This set of technologies bridge business and IT effectively and can be able to evolve in a disciplined way in course of the continual business transformation journey. It received very positive feedback from the customer.

\section{Conclusions}

Facing today's highly competitive and dynamic market environment, enterprises are forced to transform their business and IT in order to sharpen their focuses, achieve their operational efficiency and improve their responsiveness to the changes, which requires a methodology to align business with IT in an evolving way. A business driven and component based business transformation methodology framework is proposed in this paper. Based on business strategy and business needs, CBM can help identify transformation opportunities, develop transformation roadmap and guide IT architecture design from business perspective. IT blueprint method provides overall IT architecture to align enterprise application with business architecture and enterprise future development. Component-based model-driven solution building 
method facilitates the process from business process to solution design and implementation. Assetization method helps enterprise reuse useful assets in the continual transformation. Several other fundamental technologies are also mentioned in this paper. This methodology framework is adopted in a real customer engagement. It's proved that such methodology is effective not only seamlessly links from business design to business processes to IT implementation, but also coordinates the individual transformations to fulfill the enterprise transformation vision. It enables the customers' continual transformation to accommodate ceaselessly changing conditions.

\section{References}

1. R.T. Pascale and J. Sternln, Your Company's Secret Change Agents, Harvard Business Review May, $72-83$ (2005).

2. S. Kapoor and K. Bhattacharya, A Technical Framework for Sense-and-Respond Business Management, IBM System Journal 44(1), 5-24 (2005).

3. R.K. Weiler, Decision Support: Overcoming Obstacles To Asset Reuse; http://www.informationweek.com/story/showArticle.jhtml?articleID $=6502407$

4. A.W. Brown, Large-scale Component-Based Development (Prentice-Hall, 2000).

5. A.W. Brown, Model-Driven Architecture: Principles and Practice, Journal of Systems and Software Modeling, 3(4), 314-327 (2004).

6. J. Magretta, Why Business Models Matter, Harvard Business Review, May, 86-92 (2002).

7. R. Veryard, Towards the Component-Based Business: Plug and Play (Springer, London, 2000).

8. S. Ramamurthy, Simplify to Succeed Optimize the Customer Franchise and Achieve Operational Scale, IBM Business Consulting Services G510-9108-00, 2003.

9. Building an Edge (January 23, 2005); http://www.ibm.com/industries/finance/bae, Volume 5, Number 6, June 22, 2004

10. J. Zhu, Z. Tian, and T. Li, Model-Driven Business Process Integration and Management: A Case Study with Bank SinoPac Regional Service Platform, IBM Journal of Research and Development 48(5/6), 649-670 (2004). 